\title{
Nuevas subjetividades e imaginarios sociales en estudiantes de primer año de educación terciaria en Concepción, Chile. Experiencias de estudios remotos en contexto de pandemia y postestallido social*
}

\author{
New Subjectivities and Social Imaginaries in First-Year College \\ Students in Concepción, Chile. Remote Studies \\ Experiences in the Context of Pandemic and Post-Social \\ Outburst
}

Andrea Aravena R.** y Manuel Antonio Baeza R. ${ }^{* * *}$

\begin{abstract}
RESUMEN
El presente artículo tiene por objeto aproximarse a la manera en que los estudiantes de primer año de educación superior en Chile piensan e imaginan su proceso de enseñanza/aprendizaje, a partir de sus expectativas y experiencia de estudios en contexto de pandemia y postestallido social. Se exploran principalmente las expectativas juveniles de los estudiantes que en 2020 ingresaron a la educación superior, sus problemas de adaptación a la vida universitaria y la experiencia de estudios a distancia. Los hallazgos dan cuenta de la emergencia de nuevas subjetividades juveniles en estudiantes universitarios y de un proceso naciente
\end{abstract} Palabras clave: imaginarios sociales, subjetividad juvenil, estudios en línea, pandemia, estallido social.

\footnotetext{
* Proyecto PI/06 UCO 1895 "Nuevas subjetividades e imaginarios sociales juveniles de la educación superior en estudiantes de primer año de la Universidad de Concepción y el Instituto Virginio Gómez".

** Antropóloga de nacionalidad chilena, doctora en Antropología social y Etnología, École del Hautes Études en Sciences Sociales, Paris. Académica Facultad de Ciencias Sociales, Universidad de Concepción, Chile. Correo: andrea.aravena@udec.cl Orcid: https://orcid.org/0000-0002-1230-3063

*** Sociólogo de nacionalidad chilena, Dr. en Sociología, Universidad de La Sorbonne Nouvelle, París III. Académico Facultad de Ciencias Sociales, Universidad de Concepción, Chile. Correo: mbaeza@udec.cl Orcid: https://orcid.org/0000-0002-2305-9844
} 
de configuración de nuevos imaginarios sociales juveniles acerca de la educación superior.

\begin{abstract}
This article is aimed at understanding how first-year college students in Chile think about and imagine their teaching/learning process, based on their expectations and study experience within the context of the pandemic and post-social outburst. It mainly explores the expectations of teens who entered college in 2020, their problems with adapting to college life, and the remote learning experience. The findings show the emergence of new youth subjectivities among college students and a budding process of configuration of new social imaginaries among teens related to higher education.
\end{abstract} Key words: social imaginaries, youth subjectivity, online studies, pandemic, social outburst. 


\section{Introducción}

El confinamiento, como modelo de control del espacio mediante las cuarentenas, conocidas a partir de la gestión de la peste en el siglo XVI, es una creación institucional propia de aquella época en que la relación íntima de las personas con la enfermedad y la muerte pasa de ser una cuestión privada a un asunto de gerenciamiento del poder estatal (Foucault, 1975). Si bien la situación que se vive actualmente no es del todo comparable, por los desafíos que el control efectivo del espacio público y privado plantea, la idea de "confinamiento", en especial para el mundo estudiantil, se ha constituido en el elemento que probablemente marque de manera durable la subjetividad de las personas jóvenes. En el contexto de la pandemia por covid-19, a poco de conocerse el primer infectado en Chile, a mediados de marzo de 2020, el Ministerio de Educación chileno decretó la suspensión de clases presenciales en establecimientos educacionales, y las universidades e institutos profesionales establecieron que todas sus clases se realizarían bajo formato virtual.

Mientras que las instituciones se apresuraron en "capacitar" a su cuerpo académico en el uso de tecnologías de la información para la educación en línea, y así poder dar inicio al semestre de manera remota, una cantidad importante de estudiantes de nivel universitario se "movilizó" a través de las redes sociales para convocar a paros estudiantiles hasta que pudiesen tener acceso no solo a los equipos necesarios, sino también a los planes de datos requeridos para la conexión a Internet. De diferentes maneras, con recursos de las propias universidades y mediante fondos concursables, entre otros, las clases arrancaron tardíamente el primer año de la pandemia en Chile, prolongándose en muchos casos hasta enero o incluso abril del año 2021. Particularmente afectados se vieron los "mechones" — chilenismo usado para referirse en el país a estudiantes de primer año de educación terciaria-, quienes no solo no vivieron los ritos de iniciación propios de esta etapa de la vida, sino que, improvisadamente, debieron adaptarse a la "vida universitaria" en confinamiento.

Adicionalmente, el término de la enseñanza media y el tránsito a la educación superior de esta cohorte estuvo fuertemente marcado por los ecos del "estallido social" iniciado en octubre de 2019 en Chile. Entre los estudiantes, la revuelta popular no fue sino el corolario de múl- 
tiples y masivas manifestaciones iniciadas en 2001, con diversos focos de intensidad durante 2006, 2011 y 2018. Después del estallido social, en noviembre del año 2019, la Asamblea Coordinadora de Estudiantes Secundarios (ACES) llamó a un "boicot" nacional de la Prueba de Selección Universitaria y a la movilización en todos los locales donde se debía rendir dicha prueba, redundando en su postergación en dos ocasiones y en la anulación de algunas de sus partes.

La investigación que sustenta los resultados de este artículo, ejecutada entre 2020 y 2021, tiene como objetivo general comprender, desde el punto de vista de discursos de estudiantes que ingresan a primer año de educación superior/educación técnico profesional, la manera en que piensan e imaginan su proceso de enseñanza-aprendizaje, a partir de sus expectativas y experiencia de estudios en contexto de pandemia y postestallido social.

\section{Antecedentes teóricos y referenciales}

Entenderemos por "imaginarios sociales" aquellas "formas de significación institucionalizadas que adopta la sociedad en el pensar, en el decir, en el hacer, en el juzgar" (Baeza, 2011, p. 33). En este campo de investigaciones, las aportaciones son múltiples y variadas, entre las cuales podemos citar a Pintos (1995), Taylor (2006), Durand (2004), Carretero (2017), Baczko (1991), Bergua (2007), Cegarra (2012), Sánchez-Capdequí (1999), Girola (2012), Aliaga (2018), Murcia (2009). Desde Castoriadis (1975) en adelante y su doble acepción de imaginarios sociales como institución simbólica de lo social a la vez que facultad instituyente, esos autores han trabajado desde el constructivismo sistémico (Pintos, por ejemplo) hasta la sociofenomenología (Girola, Baeza), pasando por acercamientos mayores con el psicoanálisis (Durand, Sánchez-Capdequí) con las visiones históricas (Baczko, Taylor). El concepto que retenemos de este debate es el de imaginarios sociales en tanto que esquemas de significación del mundo social, en este caso específico esquemas del mundo social juvenil, y a los cuales contribuyen, a su vez, nuevos "productos" subjetivos como resultado de ese mecanismo psíquico de significación que tenemos todos los seres humanos.

Abordar entonces la dimensión imaginada de lo que consideramos "realidad" supone precisar que lo imaginario puede ser definido como 
el conjunto de significaciones e imágenes mentales y visuales mediante las cuales la sociedad y las personas organizan y expresan simbólicamente su relación con el entorno (Durand, 2004). El imaginario corresponde a un elemento fundante de la sociedad, que se expresa en su universo simbólico quedando la propia sociedad definida a partir de significaciones imaginarias e instituciones sociales que, cristalizadas, forman un imaginario social instituido, asegurando su continuidad y reproducción y regulando la vida social (Castoriadis, 2007).

Por su parte, los imaginarios sociales son fuente de significación de la realidad social (Castoriadis \& Tomès, 2007), de manera que otorgan a las personas categorías que les permiten comprender los fenómenos sociales, produciendo una imagen de estabilidad en las relaciones sociales cambiantes y dando continuidad a las experiencias discontinuas (Pintos, 1995). De este modo, las personas otorgan significados múltiples a significantes diversos, que, una vez consensuados, permiten generar un orden simbólico compartido. La propia idea de "generación", lejos de remitir a una esencia, se construye social y culturalmente, de manera subjetiva e intersubjetivamente, de manera que en una sociedad determinada es posible y deseable indagar en los significados que le son atribuidos, tanto por quienes adscriben a dicha generación como por quienes la caracterizan, como resultado de una particular construcción de la realidad (Berger \& Luckman, 1993).

La subjetividad, a su vez, se asocia con la incorporación de emociones y sentimientos, al expresar ideas, pensamientos o percepciones sobre objetos, experiencias, fenómenos o personas. La subjetividad es una cualidad humana, ya que es inevitable expresar estando fuera de uno mismo pues, pues desde un punto de vista hermenéutico, lo seres humanos no pueden vivir sin interpretar, producir y atribuir significados a todo cuanto les rodea y sucede. La subjetividad humana, entendida como aquella construcción, percepción y valorización personal y parcial sobre un aspecto de la realidad o sobre sí mismo, escapa a la mera asociación sustancializada de conciencia, y refiere a la voluntad y capacidad de ser sujeto (Alonso \& Sandoval, 2012). La construcción de la subjetividad, entonces, refiere a un proceso relacional, intersubjetivo e histórico-cultural (Ibáñez, 1994; González, 2002), y, a la vez, resulta inseparable de un marco social, político y cultural, pues lo social tiene una dimensión subjetiva (Cas- 
toriadis, 1986), tal y como se refleja en el concepto de "imaginario social” (Castoriadis, 1997). En esta investigación, en plena multicrisis (Baeza, 2020), agravada por el factor pandemia, que por cierto es un escenario problemático que incluye la educación, se hace el seguimiento de subjetividades juveniles, vislumbrando a través de ellas lo que pueden ser nuevos imaginarios sociales.

Sobre el particular, se dirá como primera aproximación, por ejemplo, que Murcia (2009) ha indagado en la vida universitaria y en los imaginarios de profesores, profesoras y estudiantes universitarios, principalmente en Colombia. Sus estudios abordan la universidad como una institución social, cuyos imaginarios se construyen a partir de la vida cotidiana. La dimensión temporal o histórica que se vive en cada época sería igualmente crucial, en la medida en que "los imaginarios sociales son también configuraciones históricas (...) que se muestran en dinámicas profundas de cambios y ebulliciones, a veces imperceptibles a miradas localizadas en cortos escenarios temporales" (Murcia, 2009, p. 239). Así, los imaginarios de estudiantes sobre la universidad girarían, por un lado, en torno a la formación profesional, centrada en lo inmediato y en el proyecto de vida personal de cada persona, en una tendencia que se vincularía a las estrategias propias del mercado, con un foco individualista en la construcción de sí mismo expresada en esta etapa. Por otro, girarían en torno al papel del estudiantado en la universidad, orientado a "aprender", a "formarse como profesional" y, en pocos casos, a "formarse como persona íntegra” (Murcia, 2009, pp. 255-256).

En un estudio realizado por Molina-Chávez y Álvarez-Valdés (2017), abocado a la identificación de elementos simbólicos del imaginario social que comienza a configurarse en Chile, en medio de transformaciones profundas en el ámbito social, cultural, político y económico de los últimos 40 años, los imaginarios sociales de lo juvenil estarían marcados, por un lado, por un factor de maduración que se vincula con la transición de niño a adulto, aspirando a una mayor autonomía personal y atravesando un distanciamiento crítico de referencias de autoridad, disciplina y obediencia asociadas a la adultez; por otro, por los procesos de escolarización, orientados a la idea de "producción social", para lograr la integración a la sociedad constituyéndose como un ser productivo y, en el caso de los estudios terciarios, como una promesa 
para cumplir ciertos estándares de vida: estabilidad económica, vivir sin deudas, una buena calidad de vida, entre otras.

Para comprender los potenciales impactos de los estudios en línea en contexto de pandemia, en una generación de estudiantes de primer año de estudios terciarios y la manera en que este contexto moldea sus imaginarios, se hace necesario contextualizar sus percepciones, vivencias y opiniones en a lo menos dos hechos significativos. Por un lado, la propia generación de jóvenes a la que pertenecen y, por otro, la magnitud de los cambios que han afectado sus vidas.

El Chile de las últimas décadas ha estado atravesado por un conjunto de hechos sociales y políticos que habrían llevado a toda una generación de jóvenes a construir su subjetividad juvenil a partir de movilizaciones estudiantiles en la enseñanza media y superior. Las movilizaciones, que comienzan con el "mochilazo en 2001", se intensifican en 2005-2006 con la llamada "revolución pingüina” y alcanzan su punto culminante con la marea feminista de 2018, a la que se suma el "estallido social" de 2019. Estos procesos habrían configurado una suerte de "ruptura generacional" (Zarzuri, 2020, p. 231), en el sentido de un discurso y una épica generacional que les permite constituirse en una "generación nueva” (Zarzuri, 2020, p. 232).

Esta generación, se ha señalado, estaría impregnada de una "sensación crónica de frustración, exclusión y abuso de poder" (Ganter \& Zarzuri, 2020, p. 84) y por la "desconfianza e indignación de décadas de promesas institucionales no concretizadas" (Ganter \& Zarzuri, 2020, p. 86). Ello llevaría a esta juventud a cuestionar profundamente el mundo adulto y sus valores, anulando el peso simbólico de la diferencia entre generaciones y quedando arrojada a un vacío, sin otros referentes más que ellos mismos (Ganter \& Zarzuri, 2020). Parte de estos elementos constituyen lo que ha sido llamado una "nueva e hipotética identidad generacional", "entendida como una construcción subjetiva en la que los actores colectivos o individuales se van nombrando a sí mismos ('nuestra generación') y diferenciando de otras" (Álvarez-Valdés \& Garcés-Sotomayor, 2017, p. 994). Los ejes gravitantes de esta identidad generacional girarían en, por un lado, expectativas de alcanzar mayores niveles educacionales, asociadas a una exigencia social, y, por otro, en el autoconcepto de ser "una generación cómoda", vinculada a la prolongación del apoyo familiar por las exigencias de mayor edu- 
cación y por el mayor acceso al consumo (Álvarez-Valdés \& GarcésSotomayor, 2017).

Por otro lado, la construcción de subjetividades en tiempos de pandemia plantea igualmente desafíos educativos que suponen un conjunto de aprendizajes que deben ser analizados y significados a la hora de hacer una evaluación de los abruptos y radicales cambios que han afectado a las comunidades educativas (Pérez, 2020). En efecto, se ha caracterizado este complejo periodo por una serie de fenómenos como: a) la evidencia de la dimensión global del sistema mundo; b) el carácter sistémico de todos los fenómenos y procesos humanos; c) el virus como manifestación del deterioro profundo del ecosistema; d) el capitalismo, especialmente en su despiadada versión neoliberal, como modelo inapropiado; e) el poder de la infodemia y la irradiación universal de bulos y mentiras; f) la apoteosis digital; g) conciencia de la fragilidad, cambio e incertidumbre de la vida humana, a pesar del progreso de la ciencia, la técnica y la industria y; h) cambio urgente de prioridades (Pérez, 2020). Todos y cada uno de estos fenómenos estarían entonces presentes y formarían parte de los propios procesos de enseñanza-aprendizaje en los que se encuentra inmerso el mundo académico, en el que estudiantes y profesores construyen subjetividades a diario marcadas por un contexto conjugado, inédito en la historia de la educación y de sus propias trayectorias históricas.

El confinamiento masivo de la población, derivado de las medidas de protección implementadas frente al avance de la pandemia por covid-19, ha generado, sin duda, grandes cambios en prácticamente todas las áreas del comportamiento humano. La educación se ha visto fuertemente afectada y en cada nivel la presencialidad ha sido sustituida por la implementación masiva de clases en modalidad remota en tiempos de excepcionalidad. Los estudios universitarios, orientados principalmente al estudio de las ciencias, y la enseñanza técnicoprofesional, enfocada en la adquisición y desarrollo de competencias propias en una especialidad determinada (Carrillo \& Jurado, 2017, p. 141), implican no solo un entrenamiento teórico sino también la adquisición de un conjunto de habilidades prácticas (Hurtado, 2020, p. 1) que, en este contexto, no pueden ser aseguradas. En el caso de Chile, además, el cierre físico de las universidades y los centros de formación técnica y profesional ha sido particularmente prolongado. 
En mayo de 2020, la ONU y el Instituto Internacional para la Educación Superior en América Latina y el Caribe (IESALC) publicaron un análisis de los impactos, respuestas y recomendaciones sobre los efectos inmediatos de la educación superior y su relación con el covid-19. El informe expuso los impactos reales y estimados - a corto, mediano y largo plazo - de la educación en línea en los estudiantes. Según una encuesta aplicada por la UNESCO a sus directores de cátedra sobre su percepción de los problemas de los estudiantes — citada en el informe mencionado-, se estableció que sus preocupaciones irían en el siguiente orden y de mayor a menor: conexión a Internet, preocupaciones financieras, regulación del estudio, equipamiento, ansiedad respecto de la pandemia, aislamiento social y problemas en la comunicación con pares y profesores.

Otro de los ámbitos aludidos se relaciona con la desigualdad social entre los jóvenes estudiantes, que se manifiesta a través de la disponibilidad de equipos técnicos que permitan trabajar de manera adecuada en la modalidad remota y, por otra, la posibilidad y accesibilidad a tener adecuadas conexiones a Internet. Estos elementos irán configurando diferentes experiencias y vivencias de aprendizaje y proyección personal y profesional. De hecho, hay quienes han destacado que, en este contexto, la disponibilidad y calidad de recursos tecnológicos para acceder a los estudios puede llegar a ser un problema a la hora de la adaptación del estudiantado a los estudios en línea, pues, para quienes tienen dificultades de acceso a Internet o no cuentan con dispositivos personales adecuados o suficientes, el trabajo y el aprendizaje pueden verse obstaculizados (Gagliardi, 2020, p. 4).

En un estudio realizado en España sobre el covid-19 y la transformación radical de la digitalización en las instituciones universitarias, Cabero-Almenara y Llorente-Cejudo (2020) abordaron el sistema de aprendizaje forzado al que tuvieron que recurrir las instituciones de educación superior, mediado por la tecnología, los efectos en la educación superior, desigualdades y brechas digitales ante la pandemia. Según los autores, los problemas con la nueva modalidad virtual comenzaron en respuesta a la dependencia de tecnologías e Internet que exige hoy la enseñanza y el estudio universitario, y cómo estos son percibidos por docentes y estudiantes. Señalan que el problema no solo radica en el uso de la tecnología, sino también en la concepción del 
aprendizaje, cuya enseñanza estaría enfocada más en los contenidos de la materia que en los estudiantes (Cabero-Almenara \& LlorenteCejudo, 2020).

Ordorika (2020) asegura que los estudios en línea, cuya preparación fue prematura e insuficiente, incrementaron los procesos de exclusión y marginación de ciertos estudiantes, profundizándose en el escenario actual, principalmente debido al desigual acceso a Internet de los hogares en América Latina, directamente relacionado con las desigualdades de las condiciones socioeconómicas. También, la condición de ruralidad y lejanía de ciertas zonas de conectividad produce una particular situación de confinamiento en el aislamiento, esta vez de carácter digital.

También se han estudiado las repercusiones en la salud mental de estudiantes de nivel universitario. Por ejemplo, un estudio de las percepciones de 74 estudiantes de la Pontificia Universidad Católica del Perú, divididos en un primer grupo con adecuados recursos tecnológicos, y un segundo carente de tecnologías de calidad, advirtió que la principal consecuencia de las clases no presenciales para el primer grupo era el estrés derivado de la sobrecarga académica, y para el segundo el estrés, la frustración y la deserción universitaria (Lovón \& Cisneros, 2020).

Sobre el particular, diversos estudios de larga data han demostrado la prevalencia de problemas emocionales en la población chilena (Vicente, 2002) y se ha sostenido que entre los grupos de mayor riesgo se encuentran las personas jóvenes (INJUV, 2021). Respecto de estudiantes del nivel terciario, se reconoce igualmente una expansión y masificación de la educación, ampliando la cobertura a los primeros quintiles socioeconómicos y a estudiantes de menor rendimiento en la enseñanza media, configurando un nuevo tipo de estudiante con tasas significativas de trastornos adaptativos, del ánimo y de ansiedad, y de desórdenes de personalidad y antecedentes de conducta suicida (Micin \& Bagladi, 2011).

Antes de la pandemia, la Primera Encuesta Nacional de Salud Mental Universitaria reconocía que un $46 \%$ del estudiantado tenía síntomas depresivos y muestras de ansiedad, y que un $54 \%$ sufría de estrés. Conforme ha señalado Barrera (2018), "la universidad es un factor 
desencadenante, que estresa a los jóvenes, pero también ellos tienen responsabilidad, porque no hay una estructura en cuanto a hábitos de estudio, de sueño o de alimentación" (Barrera, 2018, s/n). En contexto de pandemia, un estudio de la Universidad de Chile ha demostrado que el cansancio, el miedo, la tristeza y la angustia son sentimientos que dominarían la experiencia de las generaciones jóvenes e intermedias, y se establece que el factor generacional, el género y el estrato social de pertenencia serían variables importantes a la hora de definir estos problemas (Universidad de Chile, 2021).

¿Cómo se construye la subjetividad de las personas estudiantes en este contexto? ¿Se sienten preparadas? ¿Cómo viven los estudios por Internet? ¿Cómo ha sido su adaptación a la vida universitaria en este contexto? ¿Qué expectativas tienen acerca de la educación superior? Estas son algunas de las preguntas que guiaron esta investigación.

\section{Aspectos metodológicos}

La investigación que sustentó el desarrollo de este artículo fue de tipo cualitativa, con énfasis en un estudio de caso en dos centros de educación superior, diferenciados y representativos de la estratificación y segmentación de la educación superior en Chile. El estudio se desarrolló entre 2020 y 2021 en la ciudad de Concepción, capital regional de la segunda mayor región del país, ubicada en la zona centro sur, a 500 km de la Región Metropolitana de Santiago, conocida por ser una ciudad universitaria de alta complejidad. Se trabajó con las cohortes que ingresaron el año 2020 a la Universidad de Concepción (UDEC) y al Instituto Profesional Virginio Gómez (IPVG), porque ambas instituciones "atraen" a diversos tipos de estudiantes. La UDEC selecciona a los estudiantes de mayor puntaje en la Prueba de Selección Universitaria (PSU) a nivel regional, provenientes de colegios particulares (subvencionados o pagados) y de liceos municipales que imparten educación media científico-humanista, mientras que el IPVG matricula a estudiantes que optan por carreras más cortas y que provienen de escuelas municipales y liceos de educación media técnico-profesional.

La recolección de información se efectuó a través de 58 entrevistas en profundidad, realizadas mediante reuniones virtuales en modalidad en línea (vía TEAMS o ZOOM). La selección de los casos a entrevistar se hizo mediante muestreo simple, aleatorio en una primera fase y 
por cuotas en una segunda ocasión, de entre las nóminas de estudiantes de primer año de ambas instituciones educacionales. La aleatoriedad de la primera fase consistió en enviar correos electrónicos a los estudiantes y en seleccionar los casos en que éstos respondieron. La selección incluyó a estudiantes por cuotas de entre las distintas facultades y carreras, con el fin de no concentrar las entrevistas, y evitando hacer más de cuatro entrevistas a un mismo grupo de estudiantes de una misma carrera.

Para el procesamiento de la información se recurrió a la grabación y posterior transcripción de las entrevistas, que fueron codificadas con un identificador de sexo, actividad ocupacional, tipo de establecimiento donde cursó la enseñanza media, carrera y casa de estudios de la persona entrevistada. Las transcripciones fueron tratadas mediante el Software NVivo 11, con el que se construyó una malla temática a partir de la revisión y agrupación de la información de los corpus de todas ellas. Desde dichos corpus se conformaron nodos de información que fueron incorporados a sacos semánticos para fines de interpretación.

La información fue posteriormente objeto de análisis de contenido y luego hermenéutico, a partir de codificaciones grupales realizadas por el equipo del proyecto. Este trabajo estuvo orientado a validar y dar consistencia a la relación de ideas que se había extraído de los relatos. El paso siguiente fue la agrupación de las ideas en grandes grupos o metacategorías (3). A su vez, cada metacategoría fue dividida, arrojando 14 categorías que, a su vez, se abrieron en 51 subcategorías. A continuación, se presentan los resultados de las tres metacategorías agregadas en dos: por un lado, el contexto actual de estudios postestallido social y de manera remota y, por otro, las expectativas juveniles acerca de la educación superior.

\section{Principales hallazgos}

\section{El contexto de estudios postestallido social y estudios en línea}

\subsection{Experiencias en torno la complejidad política "postestallido social"}

El aumento de la conflictividad social a partir de 2011 en Chile, acompañado de una escalada de movilizaciones estudiantiles cuyo objeto 
ha sido un profundo cuestionamiento a la idea de "educación de mercado", en lo que se ha llegado a llamar el "mayo chileno" (Penaglia \& Mejías, 2019), da cuenta de una realidad que suponemos ha impactado de manera importante a un conjunto de estudiantes de educación superior en el país. Por un lado, existe un cuestionamiento al costo y la calidad de la educación en sus diferentes niveles, bajo la premisa que la educación de calidad debiese ser un derecho, en una generación que viene sosteniendo que la educación gratuita es de mala calidad y que la educación privada es de buena calidad, reflejado en los índices y rankings de rendimiento académico.

En este sentido, el "estallido social" del 18 de octubre de 2019 marcó un punto de inflexión para muchos estudiantes en Chile, pues puede considerarse un hito histórico y social que influyó en su modo de pensar y proyectar la sociedad a partir de sus experiencias. Sobre la base de las respuestas a la pregunta sobre el contexto político y social en comento, se pueden inferir dos tendencias.

Una primera se relaciona con el aislamiento individual, la prioridad en el estudio y la autorrealización, vinculada a intereses personales del proceso de enseñanza, en los que la coyuntura política nacional parece no ser un factor de relevancia para los estudiantes. Si bien se evidencia un interés en las decisiones políticas que se toman en el país, no existiría un deseo mayor de participar e involucrarse de manera directa en este proceso, más allá del plebiscito en cuestión.

Dieciocho de octubre, ¿eso de la crisis social, cierto? La verdad es que yo vivo un poco alejado del mundo, intento aislarme de esos temas (...) Yo he estado preocupado de la PSU y solo de eso, y ahora, estudiar, estudiar, nada más. (U1AGRONO1NTJA07)

(...) no me ha interesado participar de asambleas, ni en ningún contexto político (...), ni fui a muchas marchas. Estaba enfocado en mis estudios, (...) a mí me interesa estudiar mi carrera, sacarla y después estoy en la mía no más. Obviamente, igual comparto todo lo que se pide como derecho básico que todos merecemos, pero no creo que afecte mucho en mi opinión. (U1GEOLOG2NTJA23)

Una segunda tendencia se asocia a la participación activa en las movilizaciones que se produjeron durante ese periodo. La movilización social que se generó a partir del "estallido social" se identifica 
como un hito significativo en el proceso personal de algunos estudiantes.

Si bien la participación juvenil en organizaciones de carácter político sería baja, muchos entrevistados señalan haber participado como independientes de manera activa de las movilizaciones y espacios organizativos que nacieron durante este periodo, y para algunos fue su primer acercamiento a la movilización social o, bien, actualmente tienen intenciones de participar en alguna movilización. Igualmente, varios de ellos señalan que esta experiencia fue útil para reafirmar la decisión de estudiar una carrera en particular, identificándose un efecto de tipo motivacional. En otros casos, la participación y el compromiso que experimentaron durante dicho proceso se materializó en el deseo de involucrarse y participar del plebiscito para una nueva Constitución política para Chile:

Participé de conversatorios, participé de las manifestaciones, en Santiago también, en una marcha. Considero muy importante lo que se está dando desde octubre, también el proceso constituyente. Estamos trabajando por el Comando del Apruebo. Entonces, ahi tratando de ser parte del proceso, un proceso histórico y muy importante para mí. (U1CSPOLIINTJA02)

Yo espero que me influya de una manera personal, porque después de todo lo que pasó el dieciocho, mi visión como persona, como chilena, cambió mucho, porque pasaron muchas cosas que no debieron haber ocurrido. (U2BIOLOGINTJA14)

En estos estudiantes aparece igualmente una tendencia vinculada con el cambio en la percepción de la realidad que experimentaron hasta ese momento, es decir, un cambio de sus propias subjetividades estudiantiles. El "estallido social" aparece como un hecho que visibiliza no solo demandas sociales específicas, sino una desigualdad social que algunos estudiantes todavía no se habían detenido a analizar:

Cambió bastante en mi percepción de las demás personas, el tema de la institución de la PDI (Policía de Investigaciones), de carabineros, cosas así. Sobre el mismo sistema que solo reprime, sin importar en hacerle daño a los jóvenes, a los abuelos, a los niños. Antes no veía eso. (U2EDUDIF1NTJA29) 
Siento que la percepción que tenía sobre mi país cambió radicalmente. Uno, antes de eso, tenía la impresión de que había cosas malas, pero post 18 de octubre, como que esa percepción cambia (...) y ese cambio de percepción y de perspectiva de vida me motiva mucho a querer tener un rol activo en el cambio de la sociedad. (U1DERECH1NTJA11)

Siento que somos la generación que entró a la universidad después del estallido, algo súper importante (...) que siento ahora, pero en el momento no lo sentía así (...) vivía como en una burbuja y me di cuenta de que había cosas de las que no tenía ni idea. (U2DERECH3NTJA28)

Finalmente, el periodo "postestallido social" se visualiza como un escenario al que se otorga un cierto nivel de expectativas de cambio, más allá del que se ha mencionado, que se relaciona con el resultado del plebiscito. En ese sentido, estas expectativas se proyectan también en el plano universitario:

Esperaría que, después del estallido (...), que la universidad, como institución, logre establecer diálogos concretos y transparentes con su entorno, ya sea la comunidad o la región. Y que esto se vea reflejado en el contenido de las mallas académicas, las que debieran estar contextualizadas a la realidad social que vivimos. (U2ANTROP2TJA09)

Por otro lado, como fue señalado, esta generación de estudiantes fue la última en rendir la Prueba de Selección Universitaria (PSU), antes de su reemplazo por una prueba de transición, y lo hizo en un contexto histórico de revuelta popular, tornándose en una experiencia particular, no solo por ser un proceso relativamente definitorio para su futuro, sino también por el contexto de movilización social. Este estuvo marcado por sabotajes e intentos de sabotaje en distintas sedes del país, que les afectaron de distintas maneras, pero todos coinciden en describir el sentimiento de incertidumbre que les habría embargado.

Para algunos, el sabotaje de la PSU incorporó sucesos reconocidos como violentos y de poca empatía de parte de los estudiantes que promovieron la protesta, puesto que contribuyó al nerviosismo previo e intranquilidad al momento de rendir la prueba. Para otros, que pese a haber estado de acuerdo en el sabotaje, manifestaron que este suceso 
resultó ser un factor adicional al estrés que venían arrastrando desde antes de la prueba:

(...) fue complicado, porque igual hubo muchas tomas de establecimientos a nivel país. (...) Había muchas preocupaciones a la hora de dar la prueba. Y después se filtró la prueba de historia, que era para la que yo más me había preparado, fue frustrante. Yo apoyaba el movimiento, porque la PSU era una prueba segregadora (...) igual fue un poco frustrante el hecho de haberla dado, en primer lugar, pero después, que no hayamos podido terminar. Ahí sentí una tremenda frustración por el esfuerzo que había hecho de pasar por el preuniversitario. (U1DERECH1NTJA11)

Fue una constante incertidumbre, estudiar para la PSU, tras el estallido fue casi imposible; el estar en la calle, una vez el guanaco me roció con ácido, estuve herida, estuve un tiempo sin poder salir. Todo eso como que generaba la incertidumbre y nerviosismo de rendir la prueba porque igual implicaba para mí, el todo o el nada. (U2ANTROP2TJA09)

Sabotearon mi colegio, no pude rendirla tranquilo, tuve que rendirla dos veces, como que igual fue fome en ese sentido. Pero igual fue por un bien mayor, el estallido social. (U1GEOLOG2NTJA23)

\subsection{Experiencia significada de estudios remotos en contexto de pandemia}

Uno de los mayores impactos generados por la pandemia entre los universitarios, ha sido la suspensión de actividades presenciales para evitar la interacción social y el contagio, y la consecuente modificación de la vida cotidiana de los sujetos universitarios, en lo que han sido ya dos años completamente irregulares. En cuanto se comunicó la suspensión de clases presenciales, en un par de semanas todos los docentes debieron capacitarse para continuar con sus labores formativas de manera virtual. El uso de plataformas para el trabajo a distancia, entendidas inicialmente como una dificultad expresa para el cuerpo académico y especialmente para aquellos de mayor edad, debido a las brechas tecnológicas generacionales, fue acatado a partir de los recursos propios (equipos e Internet) y asumido tempranamente por profesores y profesoras. 
De acuerdo con la 9a Encuesta Nacional de Juventud, antes de la pandemia las personas jóvenes declaraban 6,4 horas diarias promedio de uso de internet (INJUV, 2019), sin evidenciar grandes diferencias por sexo y nivel socioeconómico, apreciándose diferencias de 1.17 puntos porcentuales entre jóvenes de medios urbanos frente a los de zonas rurales, y un poco más por tramo de edad: 7,24 horas en el tramo 15-19; 6,54 en el tramo 20-24; 5,67 en el tramo 25-29 (INJUV, 2019). A su vez, la mayor inversión de tiempo se dedicaba al uso de aplicaciones para chatear (83,3\% encuestados/as), descargar música (54,8\%), compartir memes (48,3\%), ver videos o series $(42,2 \%)$ y buscar información para trabajos $(37,5 \%)$, y un $24,4 \%$ señalaba que apagar el celular por algunas horas le producía ansiedad (INJUV, 2019).

Además de las diferentes posibilidades de acceso a la tecnología por parte de quienes formaron parte de este estudio, los problemas de salud mental, estrés, ansiedad y frustración se tornaron relevantes a la hora de caracterizar su primer año. En este apartado quisimos comprender cómo la suspensión de las actividades de la Universidad y el Instituto modificó sus vidas, bajo la premisa inicial de que, por ser nativos digitales, el impacto de las clases en línea podía ser menor que en otros segmentos etarios, menos acostumbrados a la exposición a las redes sociales y al mismo uso de Internet.

Sin embargo, en el contexto de la creciente desconfianza en las instituciones de la sociedad chilena, que quedó de manifiesto tras el "estallido social", pero también en el propio sistema educativo chileno desde hace décadas, el uso de tecnologías digitales como único medio para entregar la enseñanza académica a nivel terciario fue recibido con mayor desconfianza aún por parte del estudiantado.

En el caso de los estudiantes que se suponía tendrían menos dificultades para adaptarse al uso de estas plataformas (TEAMS y CAN$V A S$ en el caso de la Universidad de Concepción; ZOOM en el caso del Instituto Profesional Virginio Gómez), la adaptación al sistema fue más lento de lo esperado y no estuvo para nada exento de problemas y dificultades.

Entre los problemas más importantes que reportan las entrevistas destacan la incertidumbre acerca de la pandemia y las cuarentenas obligatorias, el miedo al contagio, a la enfermedad propia y de sus se- 
res queridos, la falta de tiempo para asistir a clases en línea y estudiar, debido a la cantidad de horas diarias dedicadas a tareas del hogar, pero, especialmente, a escuchar y ver noticias, o a procesar la cantidad de información que les llega al celular vía WhatsApp o Twitter o redes de Facebook, entre otras redes sociales. Si bien mucha de la información recibida podía estar compuesta por fake news, se reporta también la imposibilidad de discriminar entre información falsa y verdadera y, finalmente, el conjunto parece ser una tremenda cantidad de información difícil de comprender y de asimilar. Estar tan pendientes de las redes sociales, en lo que muchos describen como un "bombardeo constante" de información, provocó a estos estudiantes un tremendo problema de ansiedad, en muchos, y de estrés, en otros.

En atención a ello, a medida que avanzaba el semestre muchos entrevistados valoraban positivamente la mayor disponibilidad de tiempo para estudiar o estar con la familia.

(...) lo positivo es que dos o tres minutos antes que empiece la clase, me levanto. No es como en la $U$, que tendría que levantarme más temprano o si tuviera que ir, la demora en volverme para la casa. (U2ARQUIT2NTJA13)

Otros valoraban que la transición a la universidad se había producido de una manera menos drástica, pues el cambio había tenido que ver con nuevos contenidos y dinámicas de estudio, dejando fuera el traslado a otra ciudad o al hecho de asumir una vida alejada de la familia.

(...) siento que tuvo su parte positiva, porque cuando uno entra a la universidad es un cambio muy grande, que es como el salir de la casa y el cambio de método de estudio con mucha carga. Son dos cambios muy grandes que se viven siempre juntos, pero a nosotros nos tocó la suerte que lo vivimos separados. Solo nos tocó la densidad de la materia y la cantidad, pero no nos fuimos de la casa. Y eso para mí es un paso ganado. (U1DERECH1NTJA11)

La modalidad de estudio en línea y el uso de plataformas pareciera haber facilitado el proceso de enseñanza para algunos estudiantes. El primer argumento se relaciona con que las clases quedan grabadas en la plataforma, lo que les permite volver a revisarlas, así como la disponibilidad de material de apoyo. En el mismo sentido, las evaluacio- 
nes en esta modalidad facilitarían el uso de apuntes, pues, tal como se aprecia:

Uno puede tener el libro abierto al lado de uno, entonces, se puede ayudar y es bien dificil no pasar, hay más facilidad para pasar los cursos. (U2CSPOLI2NTJA12)

El contar con la plataforma dinámica que facilita la universidad, es un aspecto positivo. Y, por otro lado, los profes, ahora se ven de alguna manera obligados a facilitar todo el material que se tiene para las clases. Cosa que antes había que pelearlo con cada uno. Por ejemplo, PowerPoint, guías, todo queda ahí, lo que facilita el estudio. (U2ANTROP2TJA09)

Puedo ver las clases después y en una sala no sé si hubiese podido tomar apuntes, así que no sé cómo lo hubiera hecho en una clase presencial. Me ayudó también el hecho de no haber tenido que dar exámenes orales, que me daban pánico. (U1DERECH1NTJA11)

La otra tendencia, en relación con aspectos positivos de la enseñanza en línea en contexto de pandemia, es aquella que identifica el apoyo entre pares y el esfuerzo de los profesores al adaptarse de buena manera a la modalidad virtual. Ambos factores resultan ser beneficiosos en un contexto de bastante complejidad:

(...) destaco el compañerismo, porque al final se dio como el espacio, considerando la situación en que estábamos, que fue complicado, que en las clases no se prendian las cámaras. Entonces, chateando nos fuimos conociendo y nos fuimos brindando apoyo. (...) de parte de los profesores, vi que muchos estaban dispuestos a aprender a usar las plataformas, a usar de la mejor manera posible. Había un gran interés por hacer las cosas bien. (U2CSPOLI1NTJA12)

Conocer a personas distintas, igual a pesar de la pandemia, los profesores han intentado hacer lo mejor, a mí me ha tocado profesoras y profesores que se ponen en el lugar del alumno en caso de alguna dificultad. Eso podría rescatar, que han sido excelentes. (U2BIOLOGINTJA14)

La misma situación se refleja en la evaluación "es o ha sido complicado", y la complicación refiere a factores como estrés, ansiedad o frustración, entre otros, que ha afectado especialmente a un amplio 
grupo de estudiantes universitarios. Entre los problemas mencionados, la conectividad parece ser uno de los más importantes. La modalidad de estudios en línea exige no solo un buen computador que soporte las plataformas desde las que se imparte la clase, sino además una conexión a Internet buena y estable. En ese sentido, las condiciones varían entre los estudiantes; sin embargo, entre los aspectos negativos más frecuentes que se mencionan aparecen justamente los problemas de conexión.

Las mayores dificultades han sido el poder conectarse, la caída del Internet. (U1CSPOLI1NTJA02)

El estudio es igual un poco complicado, porque hay que ordenarse súper bien para que a uno le vaya bien. $Y$ de repente surgen estos distractores que, a veces, no se puede, o se corta la luz, o el Internet, iba y volvía. (U1AGRONO1NTJA07)

(...) ha afectado mi motivación en la carrera, ni siquiera alcancé a tocar la U. Entonces, como que todo se desplomó. Fue desmotivante, porque uno quería conocer a los compañeros, a los profes, tener clases presenciales y, sobre todo, con los laboratorios también, porque los ramos con laboratorio no los hemos podido realizar. (U1GEOLOG2NTJA23)

Los vivo un poco mal, no me gusta, siento que igual el tema del aislamiento, tener que estar tras un computador, no me gusta. Siento que el apego, el relacionarme personalmente con alguien ayuda mucho, sobre todo cuando se trata de la educación. Y en este contexto eso ha sido algo que no se genera. (U2BIOLOGINTJA14)

Sumado a lo anterior, la modalidad remota también influiría en la participación del estudiante en clases. Como señalan la mayoría de los estudiantes, su participación sería baja o nula, debido a la vergüenza y ansiedad que les produce "abrir el micrófono" y opinar, a lo que se añade las dificultades y limitaciones que tienen las plataformas, ya que, en el momento que dos estudiantes hablan, las voces se interfieren, dificultando el entendimiento del mensaje.

Yo no hablo en las clases y, de hecho, me da pena pensar en eso, porque yo siempre era de preguntar en clases y de quedarme con los profes - en el colegio - preguntando y conversando. Ahora no hago eso, me da vergüenza hablar en clase, escuchar mi voz en la graba- 
ción, me da demasiada vergüenza. Encuentro que es súper frio todo esto, me da pena los profes porque ellos, a veces, preguntan cosas y nadie responde, entonces se vuelve un silencio incómodo. (U2DERECH3NTJA28)

Esta forma de vivir es estresante. Yo igual era una persona que salía mucho y ahora es muy complicado. Me siento muy atrapado. Y eso me hace tener ansiedad. Y es muy difícil salir de ese círculo vicioso, porque uno está en la pieza todo el día. (U1DERECH1NTJA11)

Otra tendencia que se reconoce entre los aspectos negativos son los distractores que se encuentran en el hogar, tales como la intervención de la familia, el ruido externo y la dificultad de concentrarse en una clase a través de una pantalla. También, las obligaciones en el hogar, como "ayudar en las cosas de la casa" o el cuidado de hermanos menores, son factores negativos que enfrentan los estudiantes al estar todo el día en su hogar.

Me molesta porque mi mamá ve la tele súper fuerte o mi hermano también hace bulla. Eso me afecta, porque yo me desconcentro muy rápido; no presto mucha más atención y aquí con el celular en la mano, veo Facebook, Instagram y me pierdo. (U2CSPOLI1NTJA12)

Aparece como otro elemento importante, también, la abrumadora sensación de "no estar en la universidad", básicamente producto de la ausencia de un rito de paso entre la enseñanza media y la educación terciaria. La ausencia de "mechoneo" o de recepción de los estudiantes de segundo año a los de primer año se menciona frecuentemente como una expresión de un rito que no fue y, por lo tanto, de una suerte de etapa fallida en su progresión hacia la vida adulta. La sensación de decepción, en efecto, se hizo parte del primer año universitario, pues tal como lo expresan algunos:

Uno no conoció esa vida universitaria que tanto quería conocer. (U1DERECHINTJA11)

Es que sabes que siento que estoy como en un limbo, que no estoy $\mathrm{ni}$ en la $U$, ni en el colegio. (U2DERECH3NTJA28)

De entre los dos grupos de estudiantes que formaron parte de la muestra de esta investigación (UDEC e IPVG), las mujeres se declaran más afectadas por el estrés y la angustia que les produce el "encierro" 
y las labores domésticas; quienes estudian en la Universidad se sienten más afectados y preocupados que quienes lo hacen en el Instituto, y uno de sus principales problemas es la salud mental. Pero también para quienes estudian en el IPVG, y especialmente para las mujeres, los estudios remotos les han permitido conciliar de mejor manera las responsabilidades domésticas, y especialmente maternas, con los estudios.

\section{Expectativas juveniles acerca de la educación superior}

El cuestionamiento estudiantil a la educación de mercado en Chile ha trascendido a la movilización social, traspasándose hacia las mismas expectativas juveniles de la educación superior.

Algunos estudios han señalado que la juventud chilena tendría altas expectativas acerca de la educación (INJUV, 2018) y, de hecho, se ha dicho que dicha juventud se ha movilizado activamente en las calles para que la meta de ingresar a la educación superior no se vea frustrada por falta de recursos económicos. Según la Novena Encuesta Nacional de Juventud (INJUV, 2018), sobre una muestra nacional de 9.700 casos, las personas jóvenes poseen altas expectativas educacionales: a la mayoría le gustaría alcanzar un nivel superior de educación (técnico, universitario o posgrado). Específicamente, un $13,4 \%$ quisiera alcanzar una educación técnico profesional; un $41 \%$ una carrera profesional; un $16,2 \%$ un magíster y un $13,8 \%$ un doctorado. A su vez, dicha encuesta revela que las personas jóvenes esperan que su educación les permita "desarrollarse como persona" (41,4\% del total encuestado) y "aprender más" (23,2\%), siendo éstos los dos aspectos más importantes que creen poder lograr en la vida con la educación recibida (INJUV, 2018, p. 28).

Como resultado de la investigación se obtienen dos posiciones más o menos definidas, que han sido agrupadas en dos tipos de imaginarios. Un imaginario denominado "idealista", en primer lugar, que daría cuenta de expectativas positivas respecto de lo que se puede o se quiere hacer en la vida con la educación superior:

Lo veo como una oportunidad y un privilegio. (I1INGLOG2NTJA16)

Ya teniendo un título, voy a ganar más y le voy a poder dar una mejor calidad de vida a mi familia. (I1LOGIST2TJA08) 
Pensé que sería como en las películas, que iba a ser muy independiente, con mucha elección de ramos. (U2ARQUIT2NTJA13)

En segundo lugar, identificamos un imaginario negativo o "pesimista", conforme el cual es poco o nada lo que se puede hacer en la vida con este tipo de educación:

No tenía ninguna expectativa. Salí becada y por eso entré. (IlLABDEN1TJA56)

Veo la Universidad como una obligación. Soy el primero de mi familia que llega a la universidad. (U1INGAMB2NTJA19)

Tenía el prejuicio que hay en la actualidad, referido al tema de la repercusión social porque uno no sabe si va a terminar pagando un ojo de la cara en la carrera; si realmente va a aprender; si es que va a haber enseñanza de calidad de parte de los profesores. (U1GEOLOG2NTJA41)

No pienso que la Universidad me sirva para ganarme la vida. Tal vez tenga que hacer otra cosa porque es difícil encontrar trabajo. (U2ANTROP1TJA39)

Voy a quedar endeudado y no sé si la carrera valga la pena. O sea, sí creo que vale la pena, pero no sé si voy a encontrar trabajo y poder pagar mi deuda. (U2BIOLOGINTJA14)

$\mathrm{Al}$ ser consultados acerca de la manera en que los estudiantes piensan e imaginan la universidad, en efecto, un segmento señala que la ve como un mundo de oportunidades, una manera de ascender socialmente y obtener reconocimiento. Se trataría de un imaginario idealista del futuro, presente tanto en estudiantes que ingresaron a la universidad como en aquellos que ingresaron al instituto técnico profesional; en contraste, otros responden a la pregunta a través de los temores de toda una generación por el futuro, de estudiantes que, habiendo ingresado por "presión", se sienten derrotados desde un principio. Específicamente señalan que los estudios superiores no les permitirán ascender, por lo que no esperan nada de los mismos. Entonces, ¿para qué estudiar? ¿Para qué estudiar si con esta carrera no me voy a ganar la vida? Se trataría aquí de un discurso de tipo anticipatorio negativo, más presente en estudiantes de la Universidad que del Instituto. 
En este caso estaríamos frente a un imaginario marcado por el miedo al fracaso, al endeudamiento, a la educación de mala calidad, en el que se aprecia una suerte de ausencia de pasión por el conocimiento. Lo anterior, a pesar de que la universidad es la institución en que las personas jóvenes chilenas tienen más confianza. En efecto, un $21 \%$ declara tener una alta confianza en esta institución (INJUV, 2018), siendo el porcentaje más alto de todas las instituciones consultadas. En un escenario de desconfianzas y descontentos vigente (Aravena \& Baeza, 2015), las expectativas sobre los estudios universitarios se ven limitadas a la desconfianza generalizada en las instituciones de la sociedad chilena y a las dificultades que plantea el trinomio costo/calidad/mercado. Además de la falta de expectativas, sorprende la enunciación de desconfianzas variadas sobre la misma, pues si bien se reconoce que se puede estar estudiando bien, existe preocupación por la calidad de la educación que puedan estar recibiendo, especialmente en contexto de estudios remotos. Por otro, quienes no tienen becas completas o la beca le aporta sólo un porcentaje de los aranceles anuales, expresan un temor adicional relacionado con las dudas sobre su futura inserción laboral, que eventualmente no les permitiría pagar el costo de los aranceles para los que se han endeudado. En otros casos, la universidad es vista como una mera continuidad del colegio y de alguna manera es percibida como una obligación, toda vez cuando algunas personas dicen estudiar por un imperativo de la familia y, en otros casos, por la beca obtenida. En este sentido, la obtención de la beca es claramente percibida como una oportunidad que no se puede desperdiciar y por tanto se sienten obligados/as a estudiar con gratuidad, no obstante, no siempre tienen expectativas reales sobre esa educación. Vemos aquí una respuesta anticipatoria de desconfianza, temor al fracaso y al endeudamiento.

Al profundizar en los factores negativos asociados a las expectativas juveniles respecto de la educación superior, los y las jóvenes que formaron parte de este estudio señalan con frecuencia que uno de sus problemas principales era no sentirse con la preparación adecuada para entrar a la universidad. La falta de preparación es atribuida al tipo de establecimiento escolar en que se cursó la enseñanza media, ya sea liceo municipal o colegio particular subvencionado y principalmente debido al déficit de recursos o la calidad del profesorado. Ello les lleva 
a anticipar un miedo al fracaso que muchas veces se diluye una vez estudiando:

“El problema es no saber si uno va a rendir, si uno es capaz de comprender lo que se dice en clases. Pero con el tiempo, ese miedo se fue superando" (U1AGRONO1NTJA07).

"Pensaba que iba a ser un desafío porque mi mamá me había dicho que en esta universidad los profesores son canallas" (U2BIOLOGINTJA14).

“Me imaginaba caminando por el campus, lo que no se pudo. Por otro lado, me di cuenta de que los profesores sí te ayudan, no es como pensaba ni me los habian descrito" (U2CSPOLI1NTJA12).

Entre otros factores, los temores asociados a la experiencia universitaria son atribuidos al hecho de haber "crecido en una burbuja", esto es, en un medio conocido, en el que se sentían con menores niveles de ansiedad y preocupación que la que genera la idea de entrar a la universidad. Entre los factores que afirman contribuyeron a generar esos temores y ansiedades se encuentran los dichos de sus progenitores respecto de las dificultades de la vida adulta y, especialmente, las advertencias que habrían recibido de sus profesores y profesoras: "Los profes nos metían miedo"; "Me decían que iba a ser mucho más difícil"; "No salimos de cuarto medio con esa capacidad de relacionarnos con el mundo, entonces es como lanzarnos al vacío"; "sentía miedo de fracasar".

\section{Síntesis final}

Este artículo explora, de manera descriptiva, la experiencia de estudios remotos de estudiantes de primer año de estudios superiores, en el marco de una investigación de tipo cualitativa. A partir de 58 entrevistas semiestructuradas, aplicadas vía ZOOM o TEAMS a estudiantes que cursan primer año de estudios terciarios en Chile, se analizaron y contrastaron los discursos de jóvenes de la Universidad de Concepción y de estudiantes del Instituto Profesional Virginio Gómez (región del Biobío, Chile), analizando su experiencia significada de estudios en línea y en contexto de confinamiento por pandemia, y postestallido social, así como la manera en que resignifican los procesos de enseñanza-aprendizaje y los obstáculos que encuentran. 
En ambas instituciones, la educación a distancia, en el contexto de la crisis sociosanitaria mundial, deja en evidencia distintos aspectos de las dinámicas de desigualdad económica y social que se reproducen en todo Chile en el ámbito de la educación terciaria. En los dos planteles, igualmente, se generan contrapuntos respecto del género de los entrevistados, en términos de la manera en que hombres y mujeres enfrentan y se adaptan a esta nueva realidad.

Los resultados muestran que la manera en que viven esta coyuntura y, particularmente el confinamiento y las clases virtuales, se relaciona, por un lado, con la manera en que piensan e imaginan la universidad, como un mundo de oportunidades, una manera de ascender socialmente y obtener reconocimiento (imaginario idealista e individualista), o bien, por otro, a través de los temores de toda una generación por el futuro, que, con mayores volúmenes de incertidumbre y desmotivación, enfrenta los estudios remotos con mayor dificultad $\mathrm{y}$ vive el confinamiento con mayores niveles de ansiedad. En una sociedad altamente segmentada, las proyecciones a futuro de los estudiantes dan cuenta también de desigualdades en el plano psicológico, dependiendo de sus expectativas al ingresar a los estudios superiores y de la propia experiencia vivida, a partir de sus niveles diferenciados de acceso a Internet y de los equipos disponibles en sus casas para hacer frente a la coyuntura. En este sentido, el artículo abre temas de investigación relacionados con el impacto emocional, la salud mental y su relación con su desempeño académico, que resultan potenciados por el actual contexto de pandemia y estudios remotos.

Se evidencian así diferentes estrategias y modos de adaptación a la vida universitaria que dan cuenta de a lo menos dos perfiles, entre los cuales podrían reconocerse una gama amplia de casos. En los extremos se reconoce un perfil de tipo individualista y otro más idealista, pese a que igualmente se combinan ambos tipos. Entre los dos, se despliega el proceso de adaptación académica, relacionado con el desarrollo de competencias como hábitos de estudio, internalización de normas y conocimientos, y aquel de adaptación psicológica o socioemocional asociada al manejo de la frustración y la resignificación de los acontecimientos sociales, políticos y de salud que han caracterizado a esta generación. La experiencia significada de cada uno de estos procesos pareciera depender de la preparación previa, de las condiciones del 
hogar y también de la conectividad, así como de los recursos y la disposición personal para enfrentarlos.

También, dichos perfiles interactuarían de manera estrecha con diferentes tipos posibles de imaginarios juveniles de la educación superior. En efecto, uno de éstos se orientaría a una búsqueda de autonomía personal, en un proceso de distanciamiento y diferenciación de generaciones mayores, mientras que otro, un imaginario de tipo más instrumental/productivo, se enfilaría a la integración en la sociedad adulta y como una promesa para alcanzar ciertos estándares y metas en la vida. Ambos perfiles se corresponden con configuraciones intersubjetivas del futuro, independientes de eventuales plasmaciones individuales o idealistas, estas últimas en el sentido de utopías colectivas.

Igualmente, y contrariamente a lo que se ha sostenido respecto de que estaríamos frente a toda una generación de jóvenes cuya subjetividad juvenil se ha construido sobre la base de las movilizaciones estudiantiles y la ruptura intergeneracional, en esta investigación puede verse que persisten diferentes maneras y posicionamientos en la construcción de dicha subjetividad. Nos inclinamos más a pensar que esta identidad generacional gravitaría en torno a la búsqueda de alcanzar mayores niveles educacionales, a la comodidad relativa de lo doméstico - en algunos segmentos estudiantiles-, trasladada directamente al proceso educacional, y a la esperanza de vivir en una sociedad con mayores niveles de igualdad y justicia social. Subsiste también un perfil negativo, caracterizado por altos niveles de incertidumbre que coexiste con la inexistencia de expectativas por estudios superiores, a los que no se otorga gran valor en los eventuales procesos de ascenso social. Se insinúa, en los jóvenes de enseñanza superior, una cierta cohabitación entre, por un lado, una construcción intersubjetiva propia de un imaginario de tipo "nihilista-práctico" y, por otro, una que revela cierta voluntad de constituirse como sujeto social.

Finalmente, podemos concluir señalando, de manera hipotética, que frente a los imaginarios instituidos de la educación superior en Chile aparecen nuevas subjetividades juveniles, o significaciones, acerca de la educación superior misma. En efecto, los resultados de la investigación sugieren que quienes entran hoy a la universidad esperan que ésta se ajuste mejor a las exigencias de cada uno como individuo, y que sea capaz de responder a expectativas muy diversas: 
les provea de elementos a distancia, les entregue soporte psicológico, los prepare mejor para la vida laboral. Estas nuevas subjetividades podrían ser indicadores germinales de nuevos imaginarios sociales en materia de educación superior, emergiendo una relación entre los procesos de individualización y la exigencia de una mayor adaptación de la institucionalidad de educación superior a las expectativas y necesidades individuales. Estas expectativas, sin embargo, pueden colisionar con un sistema incapaz de responder a las mismas, en un escenario en el que se confrontarían imaginarios modernizadores e individualistas de la educación versus imaginarios clásicos de educación de masa de tipo universalista.

\section{Referencias}

Aliaga, F., Maric, M. L. y Uribe, C. (eds.) (2018). Imaginarios y representaciones sociales. Estado de la investigación en Iberoamérica. Bogotá: USTA.

Alonso, J. y Sandoval, R. (2012). Sujeto social y antropología. Despliegue de subjetividad como realidad y Conocimiento. México: UNAM.

Álvarez-Valdés, C. y Garcés-Sotomayor, A. (2017). La construcción de generación en los discursos juveniles del Chile actual. Revista Latinoamericana de Ciencias Sociales, Niñez y Juventud, 15(2), 991-1004.

Aravena, A. y Baeza, M. A. (2015). Construcción socio-imaginaria de relaciones sociales. Cinta de Moebio, 53, 147-157. DOI: doi: 10.4067/S0717-554X2015000200004

Baczko, B. (1991). Los imaginarios sociales. Buenos Aires: Nueva Visión.

Baeza, M. A. (2011). Elementos básicos de una teoría fenomenológica de los imaginarios sociales. En J. Coca, J. Valero, F. Randazzo y J. L. Pintos (Coords.). Nuevas posibilidades de los imaginarios sociales (pp. 31-42). Badajoz: TREMN-CEASGA.

Baeza, M. A. (2020). Enigmas del presente. Entre el neosalvajismo y el seudo populismo. Santiago: RIL Editores.

Barrera, A. (2018). Primera Encuesta Nacional de Salud Mental Universitaria. Gobierno de Chile, Ministerio de Ciencia, Tecnología, Conocimiento e Innovación. Recuperado de https://www. 
explora.cl/blog/los-universitarios-no-piden-ayuda-psicologica-porque-se-asume-como-un-signo-de-debilidad/

Bergua, J.A. (2007). Lo social instituyente. Materiales para una sociología no clásica. Zaragoza: Prensas Universitarias de Zaragoza.

Berger, P. y Luckmann, T. (1993). La construcción social de la realidad. Buenos Aires: Amorrortu.

Cabrero-Almenara, J. y Llorente-Cejudo, C. (2020). Covid-19: transformación radical de la digitalización en las instituciones universitarias. Campus virtuales, 9(2), 25-34.

Carretero, E. (coord.) (2017). Repensar la socialidad en la modernidad avanzada. Barcelona: Anthropos.

Carrillo, O. y Jurado, P. (2017). La educación técnico profesional y las competencias para la ciudadanía. el caso de las comunas de la Provincia de Concepción, Chile. Calidad en la educación [online], (46), 133-164. DOI: http://dx.doi.org/10.4067/ S0718-45652017000100133.

Castoriadis, C. (1986). Los dominios del hombre. Las encrucijadas del laberinto. Barcelona: Gedisa.

Castoriadis, C. (1997). El Imaginario Social Instituyente. Zona Erógena, (35), Buenos Aires.

Castoriadis, C. (2007). La institución imaginaria de la sociedad. Buenos Aires: Tusquets.

Castoriadis, C. \& Tomès, A. (2007). L'imaginaire comme tel. Paris: Hermann.

Cegarra, J. (2012). Fundamentos teórico-epistemológicos de los imaginarios sociales. Cinta de Moebio, (43), 1-13. DOI: doi: 10.4067/S0717-554X2012000100001

Durand, G. (2004). Las estructuras antropológicas del imaginario. Introducción a la arquetipología general. México: FCE.

Foucault, M. (1975). Surveiller et punir: Naissance de la prison (Bibliothèque des Histoires). París: Gallimard.

Ganter, R. y Zarzuri, R. (2020). Rapsodia para una revuelta social: Retazos narrativos y expresiones generacionales del 18-O en el Chile Actual. Universum, 35(1), 74-103.

Gagliardi, V. (2020). Desafíos educativos en tiempos de pandemia. Question/Cuestión, (1), 1-6, e312. DOI: https://doi. org/10.24215/16696581e312 
Girola, L. (2012). Representaciones e imaginarios sociales. Tendencias recientes de la investigación. En E. De la Garza y G. Leyva G. (eds.). Tratado de metodología de las ciencias sociales: perspectivas actuales (pp. 441-468). Ciudad de México: Fondo de Cultura Económica.

González, F. (2002). Sujeto y subjetividad: una aproximación históricocultural. México D F: Thomson.

Hurtado, F. (2020). La educación en tiempos de pandemia: los desafíos de la escuela del siglo XXI. CIEG, Revista arbitrada del Centro de Investigación y Estudios Gerenciales (Barquisimeto - Venezuela), (44), 176-187.

Ibáñez, T. (1994). La construcción del conocimiento desde una perspectiva sociocontruccionista. Conocimiento, Realidad $e$ Ideología, Caracas, AVEPSO.

IESALC, UNESCO. (2020). COVID-19 y educación superior: De los efectos inmediatos al día después. Recuperado de http:// www.iesalc.unesco.org/wp-content/uploads/2020/05/COVID-19-ES-130520.pdf

Instituto Nacional de la Juventud, INJUV. (2019). 9a Encuesta Nacional de Juventud, 2018. Santiago: Gobierno de Chile.

Instituto Nacional de la Juventud, INJUV. (2021). Documento Técnico. Ansiedad, Angustia, Depresión y Suicidio. Hablemos de Todo 2020-2021. Recuperado de https://hablemosdetodo. injuv.gob.cl/wp-content/uploads/2021/02/AADS.pdf

Lovón, M. y Cisneros, S. (2020). Repercusiones de las clases virtuales en los estudiantes universitarios en el contexto de la cuarentena por COVID-19: El caso de la PUCP. Propósitos y Representaciones, 8(SPE3), e588. DOI: http://dx.doi.org/10.20511/ pyr2020.v8nSPE3.588

Micin, S. y Bagladi, V. (2011). Salud mental en estudiantes universitarios: incidencia de psicopatología y antecedentes de conducta suicida en población que acude a un servicio de salud estudiantil. Terapia psicológica, 29(1), 53-64. DOI: https:// dx.doi.org/10.4067/S0718-48082011000100006

Molina-Chávez, W. y Álvarez-Valdés, C. (2017). Imaginarios sociales sobre lo juvenil en el Chile contemporáneo, Revista Latinoamericana de Ciencias Sociales, Niñez y Juventud, 85-100.

Murcia, N. (2009). Vida universitaria e imaginarios: posibilidad en definición de políticas sobre educación superior, Revista La- 
tinoamericana de Ciencias Sociales, Niñez y Juventud, 7(1), 235-266. Recuperado de https://www.redalyc.org/articulo. oa?id=773/77307110

Ordorika, I. (2020). Pandemia y educación superior. Revista de la Educación Superior, 49(194), 1-8.

Penaglia, F. y Mejías, S. (2019). El conflicto estudiantil chileno y sus efectos políticos. Polis, 15(2), 7-38. Epub 11 de septiembre de 2020. DOI: https://doi.org/10.24275/uam/izt/dcsh/ polis/2019v15n2/penaglia

Pérez, A. (2020). Los desafíos educativos en tiempos de pandemias: ayudar a construir la compleja subjetividad compartida de los seres humanos. Praxis Educativa, 24(3), 1-24. DOI: doi: https://doi.org/10.19137/praxiseducativa-2020-240302

Pintos, J. L. (1995). Los imaginarios sociales. La nueva construcción de la realidad social. Maliaño, España: Sal Terrae.

Sánchez-Capdequí, C. (1999). Imaginación y sociedad: uma hermenêutica creativa de la cultura. Madrid: Tecnos.

Taylor, Ch. (2006). Imaginarios sociales modernos. Barcelona: Paidós.

Universidad de Chile. (2021). Vida en pandemia: para un aprendizaje social de impactos y respuestas a la Crisis en la vida cotidiana. Estudio longitudinal sobre la vida cotidiana en la crisis del covid-19. Primeros resultados. Informe 1 b. Julio a diciembre de 2020. Santiago, Chile. Recuperado de https:// www.uchile.cl/noticias/166860/sectores-pobres-jovenes-ymujeres-los-mas-golpeados-por-la-pandemia

Vicente P., B., Rioseco, P., Saldivia, S., Kohn, R. y Torres, S. (2002). Estudio chileno de prevalencia de patología psiquiátrica (DSMIII-R/CIDI) (ECPP). Revista Médica de Chile, 130(5).

Zarzuri, R. (2020). El malestar de los jóvenes chilenos en el siglo XXI. De la "Revolución pingüina" al "18-O". En G. De la Fuente y D. Mlynarz (Eds.). El pueblo en movimiento: Del malestar al estallido (pp. 228-246). Santiago de Chile: Catalonia. 